\title{
TRANSLATIONS AS GAUGE TRANSFORMATIONS
}

\author{
P. K. SMRZ
}

(Received 27 April 1976)

\begin{abstract}
A local description of space and time in which translations are included in the group of gauge transformations is studied using the formalism of fibre bundles. It is shown that the flat Minkowski space-time may be obtained from a non-flat connection in a de Sitter structured fibre bundle by choosing at least two different cross-sections. The interaction terms in the covariant derivative of a Dirac wave function that correspond to translations may be interpreted as the mass term of the Dirac equation, and then the two crosssections (gauges) correspond to the description of a fermion and antifermion respectively.
\end{abstract}

\section{Introduction}

In 1955 Utiyama [6] proposed an interpretation of the gravitational field as a gauge field corresponding to the Lorentz group of transformations. In 1961 Kibble [1] suggested that translations should be included in the gauge group. In the present paper we shall analyse inclusion of translations in the gauge group using the formalism of fibre bundles [2] which was found to fit the classical gauge field theory so well [5], [3].

Structure of the space-time manifold of general relativity is contained in a connection defined in the bundle of linear frames of the manifold. The connection is reducible to the Lorentz group structure so that it determines a Lorentz type metric in the manifold, but may include torsion as well. Physically, the connection is investigated by constructing a smooth cross-section in the bundle of frames (that is, assigning a basis of the tangent vector space for each point of a region of the manifold), and then comparing it with the parallel displacements defined by the connection. As we proceed along a curve in the space-time manifold (the base manifold of the fibre bundle), the amount of Lorentz rotations the frames of the cross-section undergo is judged by comparing two curves in the bundle of frames: one following the cross-section, the other being the horizontal lift of the curve in the space-time manifold. What we measure is thus a departure from parallelism. The fundamental idea discussed in the present paper is that the translations, that is, any physical change of the space-time coordinates, may themselves be just a manifestation of a departure from parallelism in a more general fibre bundle with an enlarged 
structure group. Thus the physical space-time measurements correspond to the group operations in a fibre bundle with a 10-dimensional structure group (including 6-dimensional Lorentz group and 4-dimensional translations, but not necessarily being the Poincaré group). The base manifold of such a fibre bundle may be more general than the space-time, perhaps having more than four dimensions. The main difference between the two descriptions shows up when the flat Minkowski space-time is discussed. In the classical Lorentz structure approach the flat spacetime corresponds to the most trivial case of a flat connection, in which case a horizontal cross-section in the bundle of frames exists. When translations are included in the vertical structure, the most trivial case of a flat connection with a horizontal cross-section corresponds to a case where no space-time intervals can be detected, while the flat space-time is described by a non-trivial case.

In Section 2 the classical gauge field theory is briefly reviewed; while Section 3 gives an exact mathematical formulation to the above discussion concerning translations. In Section 4 description of the flat space-time is considered, as well as the covariant derivative of a Dirac wave function.

It should be mentioned that no global properties are investigated in the present paper, and that the word "cross-section" always means only a local cross-section defined in a neighbourhood of a point.

\section{Classical Gauge field theory}

Let $P(M, G)$ be a principal fibre bundle with the base manifold $M$ and the structure group $G$, and $E(M, F, G, P)$ be a fibre bundle associated with $P$. Notation is as in [2], $F$ denoting the standard fibre of $E$. Thus a point in $E$ is a class of pairs $(u, \xi), u \in P, \xi \in F$, under the equivalence relation

$$
(u, \xi) \equiv\left(u g, g^{-1} \xi\right), \quad g \in G .
$$

A cross-section in $E$ is thus represented by a pair of functions: $u: M \rightarrow P$ and $\xi: M \rightarrow F$. The equivalence is

$$
(u(x), \xi(x)) \equiv\left(u(x) g(x), g^{-1}(x) \xi(x)\right),
$$

and the action of $g(x)$ on $u(x)$ and $\xi(x)$ is called gauge transformation. In physics $\xi$ has the meaning of a wave function with $F$ being a vector space chosen according to the type of the particle to be described.

A connection in $P(M, G)$ describes a gauge field, and the usual covariant derivative $\nabla_{X}$ of cross-sections in $E$ (defined in [2]) leads naturally to a covariant derivative $\tilde{\nabla}_{X}$ of the wave function $\xi$ :

$$
\nabla_{X}(u(x), \xi(x))=\left(u(x), \tilde{\nabla}_{X} \xi(x)\right) .
$$

If $X$ expressed in a local system of coordinates is $\partial / \partial x^{\mu}$ then the covariant 
derivative may be written more explicitly as

$$
\tilde{\nabla}_{X} \xi(x)=\frac{\partial \xi(x)}{\partial x^{\mu}}+\omega\left(X_{\mu}\right) \xi(x),
$$

where $\omega$ is the connection form of the connection in $P$, and $X$ is a tangent vector at $u(x) \in P$, that is tangent to the cross-section $u$ and projects to $\partial / \partial x^{\mu}$ by the canonical projection $\pi$ of $P(M, G): \pi\left(X_{\mu}\right)=\partial / \partial x^{\mu} . \omega\left(X_{\mu}\right)$ is then an $x$-dependent element of the Lie algcbra $\mathbf{G}$ of the groüp $G$, expêtessed in the appropriate representation according to the action of $G$ on $F$. In the equation of motion of the particle the term containing $\omega\left(X_{\mu}\right)$ describes the interaction of the particle with the gauge field.

The field of gravitation is described when $M$ is the space-time manifold, $P(M, G)$ the bundle of linear frames of $M$, with $G$ in general $G L(4, \mathbf{R})$. The connection in $P(M, G)$ must be, however, reducible to the Lorentz structure.

There exists an alternative description of gravitation that begins with an abstract principal fibre bundle $Q(M, L)$, where $M$ is a 4-dimensional manifold and $L$ is the Lorentz group. An embedding of $Q$ into the bundle of linear frames of the spacetime manifold is then necessary to make such an approach equivalent to the classical one. Such an embedding may be determined by a specific $\mathbf{R}^{4}$-valued 1 -form defined on $Q$ [5]. Identification of this 1 -form with the canonical form of the bundle of frames provides the embedding. We shall see in the next section that such a 1 -form is naturally present in the theory with translations included in the structure group.

\section{Gauge translations}

Let $P(M, G)$ be a principal fibre bundle with a 10-dimensional structure group $G$, which has the following properties:

(i) The Lie algebra $\mathbf{G}$ of $G$ has a form of a direct sum $\dagger$

$$
\mathbf{G}=\mathbf{L} \oplus \mathbf{T} \text {, }
$$

where $\mathbf{L}$ is the Lorentz Lie algebra and $\mathbf{T}$ is a 4-dimensional subspace of $\mathbf{G}$.

(ii) The adjoint map ad $g, g \in L$, acts on $\mathbf{T}$ as the 4-dimensional representation of the Lorentz group $L$.

If $M$ is 4-dimensional, the following construction is possible. Let $\omega$ be a connection form on $P(M, G)$ that decomposes into $\omega_{L}$ and $\omega_{T}$ according to the property (i) above. Further let $\gamma: M \rightarrow P$ be a cross-section in $P(M, G)$, and construct a subbundle $Q(M, L)$ by considering $\gamma(x) L$ as the fibre of $Q$ above $x \in M$. Due to the property (ii) $Q(M, L)$ may be considered as a subbundle of the bundle of linear frames of $M$ with $\omega_{T}$ identified as the canonical form. $\omega_{L}$ then defines a connection in the bundle of frames, obviously reducible to the Lorentz structure.

$\dagger$ Referring to the vector space structure of $\mathbf{G}$. 
Even if $M$ has more than four dimensions, it may happen that $\omega_{T}(X) \neq 0$ defines an involutive 4-dimensional distribution on $M$, hence a 4-dimensional submanifold $N$ of $M$, and then $Q(N, L)$ can be embedded into the bundle of linear frames of $N$. What we observe is just the 4-dimensional manifold $N$, as the extra dimensions "escape our notice", because for such vectors $\omega_{T}(X)$ is zero and we do not measure any translation in that direction.

This is all in line with the general philosophy outlined in Section 1. The Lorentz algebra valued connection form $\omega_{L}(X)$ has the meaning of the 'infinitesimal' Lorentz transformation we are about to make if we proceed in the direction indicated by $X$. Similarly, the canonical form of a bundle of frames has the meaning of the 'infinitesimal' translation we make when we proceed in the $\mathrm{X}$-direction. Thus the identification of the canonical form with the $T$-component of $\omega$ is just a mathematical expression for "translations being measured by a departure from parallelism".

While we are putting translations in the same category as the Lorentz transformations (or the electromagnetic gauge transformations), we should also point out the differences. While the choice of a Lorentz gauge is free at least theoretically, the translational gauge must be regarded as fixed as soon as we start talking about a space-time, because it is needed in the actual construction of the space-time. It can be argued that the physical process of measuring space-time allows only a change of the Lorentz gauge, but not the part connected with translations. After all, there are physical limitations even in constructions of certain Lorentz gauges, especially where the frames involve high velocities. Perhaps, the translational gauge freedom shows up in full only in the interior of the elementary particles, where measurements of distances and time intervals with sticks and clocks are unthinkable. In such a case, what evidence can we find of the presence of translations in the gauge group? A possible answer is in the covariant derivative.

\section{Interaction terms corresponding to translations}

The interaction term in the covariant derivative (1) is in general different from zero, unless either the chosen cross-section (gauge) is horizontal, or the type of the particle is such that the gauge group acts on the standard fibre via the trivial representation in which all its elements are mapped to the identity element (the particle is then 'neutral' with respect to the field concerned, as, for example, electro-magnetically neutral particles described by real wave functions with the gauge group $U(1)$ acting as the identity on $\mathbf{R}$ ).

In our case, the cross-section certainly must not be horizontal if it is to determine the canonical form and define a space-time manifold. Hence it is possible that at least some particles have the interaction terms corresponding to translations present in their equations of motion, and they should be there even in the flat 
space-time. The mass term in the Dirac equation can be interpreted as such an interaction term. In fact, it is enough that the covariant derivative has a form

$$
\frac{\partial \xi(x)}{\partial x^{\mu}}+m \frac{i}{2} \gamma_{k} h_{\mu}^{k}(x) \xi(x),
$$

where $\gamma_{k}, k=0,1,2,3$, are the Dirac matrices, and $h_{\mu}^{k}(x)$ are the 'tetrads' determining a relation

$$
\frac{\partial}{\partial x^{\mu}}=h_{\mu}^{k}(x) X_{k}
$$

between the Lorentz orthonormal frames $\left\{X_{k}, k=0,1,2,3\right\}$ and the natural frames $\left\{\partial / \partial x^{\mu}, \mu=0,1,2,3\right\}$ of the general coordinate system.

At the same time, $h_{\mu}^{k}(x)$ are also the coordinates of the canonical form on the specific cross-section in the bundle of frames, and the term $\omega_{T}\left(X_{\mu}\right)$ is, therefore, expected to have a form

$$
\omega_{T}\left(X_{\mu}\right)=h_{\mu}^{k}(x) P_{k},
$$

where $P_{k}, k=0,1,2,3$, are the Lie algebra elements forming $\mathbf{T}$. The covariant derivative (2) is then of the form (1) if $T$ is spanned by the elements

$$
P_{k}=m \frac{i}{2} \gamma_{k}
$$

An important point is that the $P_{k}$ 's defined in (3) do not commute. In fact, together with the generators of the Lorentz group

$$
L_{i k}=\frac{1}{4}\left(\gamma_{i} \gamma_{k}-\gamma_{k} \gamma_{i}\right)
$$

they form a de Sitter group, the $(3+2)$ de Sitter group in this particular choice of $P_{k}$. Alternatively, the generators of $\mathbf{T}$ could be chosen as $\frac{1}{2} m \gamma_{k} \gamma_{5}, \gamma_{5}=\gamma_{0} \gamma_{1} \gamma_{2} \gamma_{3}$, leading to the alternative form of the Dirac equation with mass $m$. The structure group is the $(4+1)$ de Sitter group in the latter case.

It is important to realize that, even with a de Sitter group as the structure group, the connection in the space-time manifold constructed according to Section 3 may be flat. Such a situation is illustrated by an example of a principal fibre bundle $K(K / S, S)$, where $K$ is the group $S U(2,2), S$ is a de Sitter group. $K / S$ is a 5dimensional base manifold, but the unique invariant connection together with a specific cross-section (that happens to be a subgroup of $K$ ) defines a 4-dimensional manifold with a flat linear connection. More detailed description of this example may be found in [4]. Here we shall discuss the situation in more general terms.

Let $\gamma$ be a cross-section in $P(M, G), G$ being a de Sitter group. Further, let there be a connection in $P(M, G)$. If a flat space-time is to be defined, the tangent vector 
space of the submanifold $\gamma(M)$ of $P$ must decompose at each point into a nonhorizontal 4-dimensional subspace $N_{(x)}$ and a horizontal subspace filling the remaining dimensions. Moreover, vertical parts of vectors in $N \gamma_{(x)}$ must be the fundamental vector fields generated by elements of $\mathbf{T}$ only, and finally there must exist four commuting vector fields belonging to $N \gamma_{(x)}$ that determine a local coordinate system in the 4-dimensional submanifold of $\gamma(M)$, which is then identified as the flat space-time. Let $X, Y$ be two such vector fields: $X(\gamma(x)), Y\left((\gamma(x)) \in N \gamma_{(x)}\right.$, and $[X, Y]=0$. If we decompose both $X$ and $Y$ into the horizontal and vertical components, we have

$$
X=X_{h}+X_{v}, \quad Y=Y_{h}+Y_{v},
$$

where $X_{v}$ and $Y_{v}$ are fundamental vector fields generated by elements of $\mathbf{T}$. Now $[X, Y]=0$ implies

$$
\left[X_{h}, Y_{h}\right]+\left[X_{h}, Y_{v}\right]+\left[X_{v}, Y_{h}\right]+\left[X_{v}, Y_{v}\right]=0 .
$$

When the horizontal and vertical components of (4) are considered separately we have

$$
\left[X_{h}, Y_{h}\right]_{v}+\left[X_{v}, Y_{v}\right]=0
$$

and

$$
\left[X_{h}, Y_{h}\right]_{h}+\left[X_{h}, Y_{v}\right]+\left[X_{v}, Y_{h}\right]=0 .
$$

Also $\left(X_{h}, Y_{h}\right]_{h}=0$, as

$$
\begin{aligned}
0 & =\pi\left[X_{h}+X_{v}, Y_{h}+Y_{v}\right] \\
& =\left[\pi\left(X_{h}+X_{v}\right), \pi\left(Y_{h}+Y_{v}\right)\right] \\
& =\pi\left[X_{h}, Y_{h}\right],
\end{aligned}
$$

where $\pi$ denotes the canonical projection in $P(M, G)$. In this way we have the conditions

$$
\begin{aligned}
& {\left[X_{h}, Y_{h}\right]_{v}+\left[X_{v}, Y_{v}\right]=0,} \\
& {\left[X_{h}, Y_{v}\right]+\left[X_{v}, Y_{h}\right]=0 .}
\end{aligned}
$$

As $\left[X_{v}, Y_{v}\right]$ is a fundamental vector field generated by an element of $\mathbf{L}$, condition (5a) may be satisfied only if $\left[X_{h}, Y_{h}\right]_{v}$ is of the same type, in other words the curvature form of the connection in $P(M, G)$ should be $\mathbf{L}$-valued. This condition is satisfied in the example of [4] by the invariant connection in $K(K / S, S)$. It is clear that a flat connection could never satisfy (5a).

Equations (5a) and (5b) are invariant with respect to the change $X_{v} \rightarrow-X_{v}$, $Y_{v} \rightarrow-Y_{v}$. It implies that if there is one cross-section $\gamma$ that defines a flat space-time then there is at least a second cross-section obtained by the reversal of the vertical components of the tangent vectors, and this second cross-section also defines a flat space-time. In the covariant derivative the two cross-sections lead to the two opposite signs of the mass term, corresponding to a description of a fermionantifermion pair. Such a pair can thus be considered as one particle described in 
two different gauges, both deriving a flat Minkowski space-time from a given nonflat connection in the original de Sitter structured fibre bundle. Links to the space-time reversal are also apparent.

\section{Concluding remarks}

An interesting point that invites attention is the role of the de Sitter group. Normally, whenever a de Sitter group is used in physics, the "raudius of trañiatitiuns" $R$ is very large, so that the de Sitter group is close to its contraction limit to which it passes when $R \rightarrow \infty$, namely the Poincaré group. Here, $R$ is in fact equal to $1 / m$, where $m$ is the mass of a fundamental fermion. This is a very short distance comparable with the dimensions of the particle. If the structure group is the $(4+1)$ de Sitter group, then the small range of the spatial "translations" may actually correspond to the small dimensions of the elementary particles. The following speculative consideration is presented just as a matter for thought. The macroscopic measurement of distances is done by following a fixed cross-section and comparing it continuously with the horizontal direction (see Sections 1 and 3). In this way the compact de Sitter transformations "unwind" and the result is interpreted as a measurement of a distance that may be many times larger than the range of the parameters in the de Sitter spatial "translations".

If, however, we could also follow the horizontal direction all the way (that is, not making any translation) then the 'distance' between the two points measured by the amount of the translational parameters needed to get from the end-point of the horizontal path to the end-point of the path following the cross-section could never be more than $2 \pi R$, the range of the spatial translational parameters. Thus if the interior of elementary particles is that region in which macroscopic space measurements are not possible and no cross-section is fixed, then the distance between any two points within the region could be expected to be of the order of $R=1 / \mathrm{m}$. On the other hand, as the time translations are non-compact in the $(4+1)$ de Sitter group, the time extent of such a region could be infinite, providing for the existence of stable particles. Thus the experimental fact of the small spatial extent and an infinite time extent of fundamental fermions supports the choice of the $(4+1)$ de Sitter group for the role of the structure group.

\section{References}

[1] T. W. B. Kibble, "Lorentz invariance and the gravitational field", J. Math, Phys. 2 (1961), 212-221.

[2] S. Kobayashi and K. Nomizu, Foundations of Differential Geometry, Vol. I, Interscience, New York (1963).

[3] P. Smrz, "Gauge invariant interactions of classical fields", J. Aust. Math. Soc. 19A (1975), 376-383.

[4] P. Smrz, "Space-time and connections in Lie groups", Proceedings of the International Symposium on Relativity and Unified Field Theory, Calcutta 1975-76. 
[5] A. Trautman, "Fibre bundles associated with space-time", Rep. Math. Phys. 1 (1970), 29-62.

[6] R. Utiyama, "Invariant theoretical interpretation of interaction", Phys. Rev. 101 (1955), 1597-1607.

Department of Mathematics

University of Newcastle

New South Wales 2308

Australia 